\title{
Comparing Total Hip Replacement Drug Treatments Based on Cost and Length of Stay
}

\author{
Blake James Huebner ${ }^{1}$ and Rhonda C Magel ${ }^{1 *}$ \\ ${ }^{1}$ Department of Statistics, North Dakota State University, Fargo, ND, United States \\ Email: rhonda.magel@ndsu.edu
}

\begin{abstract}
Drugs within three categories used in hip replacement surgery are compared based on their association with total hospital costs and length of stay. The drugs are also compared based on their associations with proportions of early readmissions. Multiple regression, logistic regression, chisquare tests, and multiple comparison techniques are used. The combination of warfarin and enoxaparin is associated with the highest cost and length of stay of the anticoagulants studied. There is no clear regional anesthesia drug used associated with the highest hospital cost and longest length of stay. Tranexamic acid is found to be associated with a smaller proportion of blood transfusions, a shorter length of stay, with no significant difference in cost found and no significant difference found in the proportion of early readmissions.
\end{abstract}

Keywords: anticoagulants, regional anesthesia drugs, antibribrinolytics, hospital cost, length of stay, proportions of early readmission, proportions of blood transfusions

\section{Introduction}

In a time of rising medical costs, hospital administration looks for any method to reduce overall costs to patients while maintaining the same level of care. In fact, after adjusting for inflation the average cost per stay from 1997 to 2010 increased by nearly $45 \%$ [1]. As a result of this rise in cost, there has been an increased interest in research on nurse to patient ratios, length of stay (LOS), and other methods to reduce overall cost. One research area that could be further investigated would be to evaluate classes of drugs that could be used for a particular type of surgery and compare how they affect overall cost and LOS. The objective of this research is to provide insight as to which particular drugs administered perioperatively to patients undergoing total hip replacement (THR) surgery within a Midwest hospital network contribute to a significant reduction to these two measurements. In addition to these two measurements, we will also examine and compare the proportion of early readmissions within each of the drug categories, and the proportion of patients requiring blood transfusions within each of the categories.

Total Hip replacement surgery is considered one of the most successful surgeries in all of medicine [2]. According to the Centers for Disease Control and Prevention, in 2010 more than 310,800 patients underwent this procedure in the United States [3]. The number of hip replacements has more than doubled between 2000 and 2010, and future projections indicate this surgery will continue to quickly rise [3]. The surgery involves an orthopedic surgeon first removing the diseased or damaged cartilage and bone, and then fitting an implant to restore functionality of the hip [2]. Candidates for the surgery include those with a damaged hip due to arthritis, a bone fracture, or other condition $[2,4]$ Research has been conducted on many aspects of hip replacement surgery. This research includes type of prostheses to use [5], and patient satisfaction [6]. Research has also looked at rehabilitation of these patients using yoga [7] and other techniques [8]. Studies have also suggested that mental health is also important in recovery [9]. In this study, we are only concerned about the types of drugs administered to hip replacement patients and any relationships these drugs may have to the measurements that have been already listed. There are numerous drugs administered to hip replacement patients throughout the surgical process. Within this study we will be concentrating our efforts of analysis on three types of drugs: anticoagulants, spinal blocks, and a blood transfusion prevention drug (an antifibrinolytic).

First, we analyze the different combinations of anticoagulants administered to the patients. Administering anticoagulants is one approach to preventing a complication called deep vein thrombosis 
(DVT) associated with THR surgery. According to the American Academy of Orthopaedic Surgeons (AAOS) [10], DVT is one of the greatest risks associated with this type of surgery. DVT is the formation of a blood clot within a deep vein, commonly in the lower extremities such as the thigh or calf. DVT can lead to impairment, or if the clot reaches the lungs, pulmonary embolism (PE) which can be fatal [11]. Treatments for the prevention of DVT fall into one of three categories including the following: compression elastic stockings and medical devices, movement and rehabilitation, and anticoagulant therapy. This research examines the effects of antigoagulant therapy only associated with the total hospital cost and LOS. Anticoagulants are typically administered the night before surgery and continue well into the postoperative care. The AAOS [10] states, "Without this preventive treatment, as many as 80 percent of orthopaedic surgical patients would develop DVT, and 10 percent to 20 percent would develop PE."

Second, we analyze different regional anesthetics administered to the patient. There are various drugs that can be given to the patient in this category and we will test for any association between the different drugs in this category and total hospital costs, and then LOS.

The last drug type we analyze is antifibrinolytics. In this study we focus on one drug of this type which is called tranexamic acid (TXA). Perioperative bleeding is a major concern for THR and is associated with not only higher risks, but also higher costs to the patients [12]. The objective of TXA is to reduce bleeding and, therefore, reduce the risks associated with blood loss which include receiving a blood transfusion [12]. There has recently been an increase in the interest of using antifibrionlytics during orthopedic surgery, and the efficacy as well as possible side effects are still being studied, which will be discussed later.

Although the complications and efficacy of the drugs are discussed, the main goal of this study is to analyze drug combinations which may result in lower overall hospital cost and LOS while maintaining the same re-admission rate. We are provided with an existing data set of total hip replacement patients and compare costs and LOS of different anticoagulants administered. We then perform similar analyses on different regional anesthetics, and a blood transfusion prevention drug. Afterwards, a recommendation is made as to which drugs tend to result in a minimal cost and LOS. The proportions of early readmissions and the proportions requiring blood transfusions within each of the drug categories will also be studied.

\section{$2 \quad$ Literature Review}

Extensive research has been done on each drug within this study in terms of medical testing. Traits of the drugs such as efficacy, possible side effects, and other medical interests have been studied. While less common, there has also been research done on a few of these drugs in relation to length of stay and costs to the patient. It is our intention to highlight important aspects and studies of these drugs in order to build a better understanding. It is important to note this research does not acknowledge or compare different dosages levels or drug delivery systems.

As mentioned previously, one of the major complications associated with hip replacement surgery is the risk of developing DVT which can lead to PE. To lower the risk, many patients are prescribed an anticoagulant as a preventative measure against blood clots. However, with blood thinners there is a possibility of increased bleeding. Three anticoagulants often prescribed include: warfarin, enoxaparin, and rivaroxaban. Warfarin is the most commonly used anticoagulant for THR [10]. It has been shown that warfarin is effective against thromboembolic complications while causing minimum bleeding complications [13]. Enoxaparin and rivaroxaban are also commonly used, in place of or in addition to warfarin. Clinical studies have shown that rivaroxaban provides a lower risk of symptomatic venous thromboembolism when compared to enoxaparin; however, the risk of major bleeding was higher when using rivaroxaban as opposed to enoxaparin [14,15]. Of the three, warfarin is associated with the least risk of bleeding complications [16].

Several studies have found that using regional anesthesia compared with general anesthesia has a favorable outcome for the patient. Patients receiving regional anesthesia were less likely to develop DVT/PE, had decreased intraoperative blood loss, and were less likely to receive a blood transfusion $[17,18,19]$. Within the regional anesthesia drug category we analyze three drugs: bupivacaine, lidocaine, and ropivacaine. Lidocaine began to be used as a spinal anesthetic in 1945 and has been widely used 
ever since [20]. It is often chosen because of its rapid onset (3-5 minutes), dense blockage, and short duration of action (1-1.5 hours) [20,21]. Thus, it is recommended for short to intermediate length surgeries [20]. The largest concern associated with lidocaine is the occurrence of transient neurologic symptoms (TNS) during postoperative care [20]. TNS presents as pain in the lower back and lower extremities including the buttocks, thighs, and lower limbs after recovery from spinal anesthesia [20]. As a result of this research into TNS, lowering the concentration of lidocaine is recommended, and other spinal anesthetics have risen in popularity. Bupivacaine is one alternative that has shown to have less incidence of TNS as compared with lidocaine [21,22,23]. However, it has been found that patients using bupivacaine have significantly longer times to ambulate and to void than when using lidocaine [22]. Bupivacaine is commonly used for intermediate to long surgeries because of its onset time of 8 minutes and duration of anesthesia lasting 210 to 240 minutes [20]. Ropivacaine is the third spinal anesthetic and is relatively new compared to the others. Like bupivacaine, ropivacaine also shows a lower relative risk for developing TNS when compared with lidocaine [21]. When compared with bupivacaine, ropivacaine is shown to give patients more motor function, a faster postoperative recovery, and superior pain relief during the first 24 hours of recovery $[24,25,26]$.

Perioperative bleeding is a major concern with total hip replacement surgery as it is associated with additional risks and costs to the patient [12]. As a result of major bleeding, many patients require blood transfusions which can cause more complications and a longer LOS [27]. Recently, tranexamic acid has been more commonly used in an attempt to reduce the overall blood loss. Several studies have concluded that TXA effectively reduces blood loss and the need for transfusions while not increasing the risk of complications such as DVT $[28,29,30]$. In addition, the administration of TXA is also seen to reduce overall costs to the patient $[31,32]$.

\section{Methodology}

\subsection{Data Description}

Data on 1214 recent THR surgeries were provided by a hospital network with a total of five locations preforming the procedure. Other variables provided include: cost of hospital stay (in USD), length of stay (in days), age (in years), gender $(\mathrm{M} / \mathrm{F})$, blood transfusion $(0 / 1)$, re-admittance $(0 / 1)$, and indicator variables of drugs administered to the patient. Under blood transfusion, 0 indicates no transfusion whereas 1 indicates at least one transfusion. Under re-admittance (RA), 0 indicates no re-admittance and 1 indicates the patient needed to return due to complications within 30 days. The variable names for the indicator variables of anticoagulants and regional anesthetics are called 'acombo' and 'scombo' respectively, and the coding key can be found in Tables 3.1 and 3.2. The blood transfusion prevention drug, tranexamic acid, is called 'b1' and is coded as 0/1; 0 indicates the drug was not administered and 1 indicates the drug was administered.

Table 3.1 Key for Anticoagulants

\begin{tabular}{cl}
\hline 'acombo' & Drug Combination \\
\hline 0 & No Anticoagulant \\
1 & Warfarin \\
2 & Enoxaparin \\
3 & Rivaroxaban \\
12 & Warfarin, Enoxaparin \\
13 & Warfarin, Rivaroxaban \\
23 & Enoxaparin, Rivaroxaban \\
\hline
\end{tabular}

Table 3.2 Key for Regional Anesthetic

\begin{tabular}{cl}
\hline 'scombo' & Drug Combination \\
\hline 0 & No Regional Anesthetic \\
1 & Lidocaine \\
2 & Bupivacaine \\
3 & Ropivacaine \\
12 & Lidocaine, Bupivacaine \\
13 & Lidocaine, Ropivacaine \\
23 & Bupivacaine, Ropivacaine \\
123 & All Three Drugs \\
\hline
\end{tabular}




\subsection{Analysis}

An Ordinary Least Squares (OLS) Regression is conducted to determine if a relationship between cost of hospital stay and drugs administered exists [33]. The first model will be formed with the dependent variable being cost, and the following independent variables: age, gender, and an indicator variable of which anticoagulant drugs were administered. If age and gender are insignificant they will be removed from the model. A similar example using backward regression techniques is given in [34] to predict weight among children based on various other variables including race, gender, age, and height, among others (pages 403-422). Residual analysis will then be performed in order to check OLS assumptions. If the assumptions of equal variance or normality of residuals is violated, a natural log transformation will be applied to the dependent variable. In addition, outliers will be located and removed based on influence. After these steps are completed the final model will be used to form conclusions on whether or not anticoagulants have a significant association with cost. If there is a difference in mean total cost associated with the different anticoagulants, multiple comparison testing using Tukey's test and Dunnett's test will be done to determine which anticoagulants have significantly smaller/ larger mean total costs associated with them. A use of multiple comparison using Tukey's test and Dunnett's test may be found in [35] on pages 98-101. This same overall procedure will be used again by replacing anticoagulants with spinal blocks and again with a blood transfusion prevention drug.

The same procedure will be followed again by replacing cost with LOS as the dependent variable. An OLS regression model will be formed followed by residual analysis, a natural log transformation, if necessary, and multiple comparison testing. Again, significant outliers may be removed based on influence. This procedure will be used separately for all three drug categories.

A test will be conducted to determine if one or more of the anticoagulants had a significantly higher proportion of re-admittance, RA, within 30 days. A chi-square test will be used, and if chi-square assumptions are not met, Fisher's exact test will be performed. A use of a chi-square test comparing proportions may be found in [36] comparing the proportion of head injuries between those individuals wearing a helmet versus those not wearing a helmet when a sample of 793 bicycle accidents is considered (pages 342- 347). An example of Fisher's exact test may be found in [37], pages 120-122. A logistic regression model will be developed to test whether anticoagulants have an effect on RA while controlling for age and gender. If they do have an effect, the model will estimate the probability of a re-admittance based on the anticoagulant used. A similar procedure will be followed as in the low birth weight study, the prostate cancer study, and the ICU study given in [38]. This same overall procedure will be used again by replacing anticoagulants with spinal blocks and again with the blood transfusion prevention drug. When analyzing the blood transfusion drug, a chi-square test will be performed to see if the drug effectively reduces the probability of receiving a transfusion. A chi-square test will also be used to explore the relationship between blood transfusions and RA, as well as blood transfusions and gender.

If the drug combinations administered are confounded upon location, further analysis will be performed, so that results obtained will be controlled for location. A location with both large sample size and adequate variability of drugs administered will be chosen. A duplicate analysis, as previously described, will be performed on drugs used at this chosen location.

\section{$4 \quad$ Results}

\subsection{Dependent Variables}

The data set consists of information on 1214 THR surgeries performed. The average cost of a hospital stay for a patient in this sample was $\$ 10,975$ with a standard deviation of $\$ 2,846$. The average length of stay (LOS) for a patient was 2.76 days with a standard deviation of 1.31 days. There were 33 patients who were readmitted to the hospital within 30 days of the procedure, or $2.72 \%$ of the patients in this sample were readmitted. 


\subsection{Anticoagulants}

We will begin by analyzing anticoagulants in terms of hospital cost, LOS, and early readmissions (RA). The number of patients and percentage of total patients using the various anticoagulants are given in Table 4.1. The average cost and the standard deviation of costs of patients using each of the anticoagulants are given in Table 4.2 .

Figure 4.1 contains box plots of cost by each acombo. The outside bounds of each of the boxes represent the 25 th and 75 th percentiles. The inner line represents the median, and the diamond represents the mean. All outer dots represent outliers. There were three extremely large outliers removed from the sample before any means were calculated. A regression analysis is conducted with the hospital cost as the dependent variable. The independent variables include the indicator variables for the different anticoagulants (acombo), gender of the patient, and age of the patient. Since acombo groups 13 and 23 had only a small number of observations, the observations associated with them were removed from the model before conducting a regression analysis. Hence, there were four indicator variables representing the various anticoagulants. Gender and age were found to be insignificant in the model and hence removed. Based on examining the box plots in Figure 4.1, a natural log transformation was conducted on the dependent variable of total hospital cost since it appears that the assumption of equal variance may be violated. The regression model output with natural log of cost as the dependent variable and the indicator variables for the anticoagulants as the only independent variables is given in Table 4.3. The large $\mathrm{F}$ value indicates that there is a significant association between hospital cost and the different anticoagulants. A multiple comparison was conducted on the various anticoagulants using Tukey's test. The comparisons found to be significant, along with $95 \%$ confidence intervals on the differences in costs, are given in Table 4.4. The results based on the transformed data are given in part a. Since the untransformed results are easier to work with and the same significant differences were found, these results are given in part b. There is no significant difference in costs between anticoagulants 12 and 1 and then between anticoagulants 0,2 , and 3 . Anticoagulants 12 and 1 are associated with the highest costs and 0,2 , and 3 with the lowest costs.

A histogram of the residuals with the dependent variable of natural log of total hospital cost is given in Figure 4.2. The residuals appear to be approximately normally distributed. Figure 4.3 gives the new set of box plots. It appears that the variances are approximately equal.

Table 4.1 Anticoagulant Frequency Table

\begin{tabular}{ccccc}
\hline Acombo & Frequency & Percent & $\begin{array}{c}\text { Cumulative } \\
\text { Frequency }\end{array}$ & $\begin{array}{c}\text { Cumulative } \\
\text { Percent }\end{array}$ \\
\hline 0 & 137 & 11.29 & 137 & 11.29 \\
1 & 463 & 38.14 & 600 & 49.42 \\
2 & 258 & 21.25 & 858 & 70.68 \\
3 & 265 & 21.83 & 1123 & 92.50 \\
12 & 77 & 6.34 & 1200 & 98.85 \\
13 & 3 & 0.25 & 1203 & 99.09 \\
23 & 11 & 0.91 & 1214 & 100.00 \\
\hline
\end{tabular}

Table 4.2 Cost Summary Statistics by Acombo

\begin{tabular}{ccc}
\hline Acombo & Mean & $\begin{array}{c}\text { Standard } \\
\text { Deviation }\end{array}$ \\
\hline 0 & $\$ 10,027.06$ & $\$ 1,849.68$ \\
1 & $\$ 11,956.46$ & $\$ 2,485.37$ \\
2 & $\$ 10,068.67$ & $\$ 1,939.50$ \\
3 & $\$ 10,023.33$ & $\$ 1,735.10$ \\
12 & $\$ 13,230.43$ & $\$ 6,591.87$ \\
13 & $\$ 10,687.45$ & $\$ 1,790.66$ \\
23 & $\$ 9,985.25$ & $\$ 1,340.83$ \\
Overall & $\$ 10,975.00$ & $\$ 2,846.00$ \\
\hline
\end{tabular}

Note: Three outliers were removed before forming this table. 
Table 4.3 Regression Model Output

\begin{tabular}{cccccc}
\hline Source & DF & Sum of Squares & Mean Square & F Value & Pr $>$ F \\
\hline Model & 4 & 8.6809 & 2.1702 & 71.23 & $<.0001$ \\
Error & 1192 & 36.3154 & 0.0305 & & \\
Corrected Total & 1196 & 44.9963 & & & \\
\hline
\end{tabular}

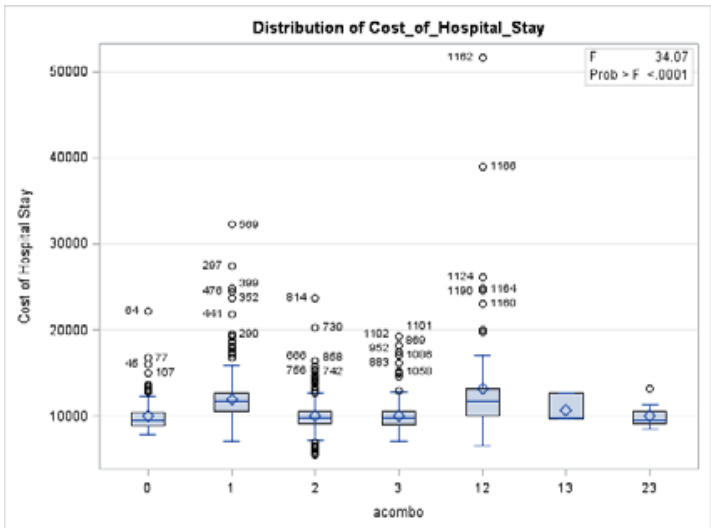

Figure 4.1 Distribution of Cost by Acombo

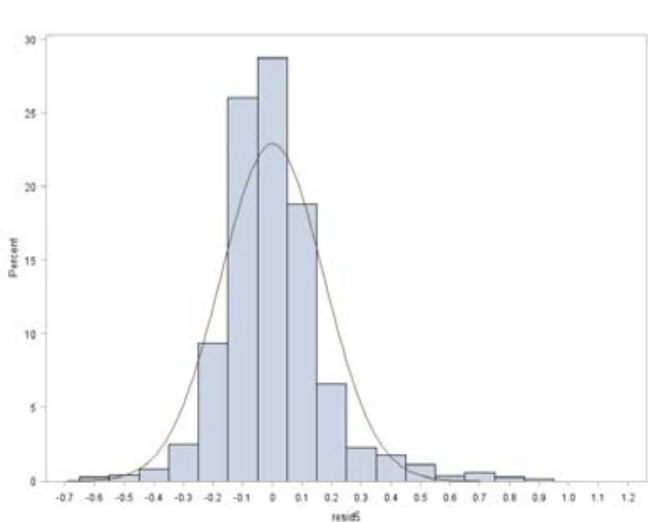

Figure 4.2 Histogram of Residual

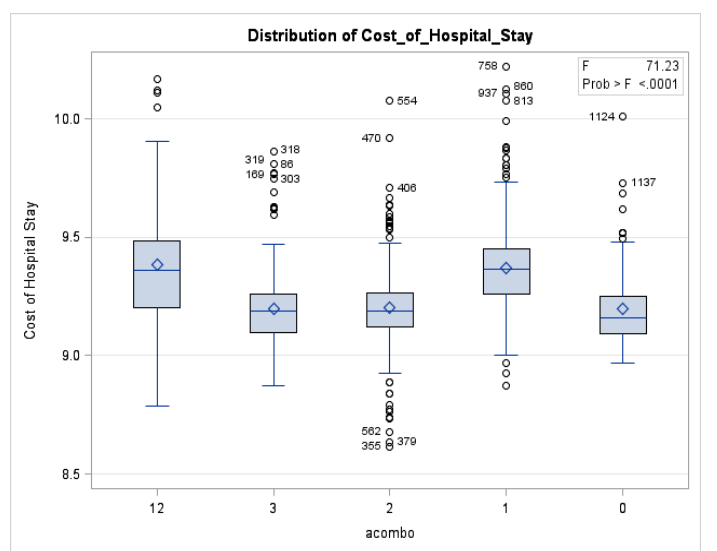

Figure 4.3 Distribution of Cost by Acombo (Natural Log of Cost)

Table 4.4(a) Significant Cost Results from Tukey-Transformed Model

\begin{tabular}{ccccc}
\hline Acombo Comparison & Percent Increase Estimate & 95\% Confidence Interval & .05 level significance \\
\hline $12-2$ & $20.06 \%$ & $12.78 \%$ & $27.81 \%$ & $* * *$ \\
$12-3$ & $20.18 \%$ & $12.91 \%$ & $27.92 \%$ & $* * *$ \\
$12-0$ & $20.22 \%$ & $12.26 \%$ & $28.74 \%$ & $* * *$ \\
$1-2$ & $18.34 \%$ & $14.04 \%$ & $22.81 \%$ & $* * *$ \\
$1-3$ & $18.46 \%$ & $14.19 \%$ & $22.89 \%$ & $* * *$ \\
$1-0$ & $18.50 \%$ & $13.13 \%$ & $24.13 \%$ & $* * *$ \\
\hline
\end{tabular}

Table 4.4(b) Significant Cost Results from Tukey-Untransformed Model

\begin{tabular}{ccccc}
\hline Acombo Comparison & Dollar Increase Estimate & 95\% Confidence Interval & .05 level significance \\
\hline $12-2$ & $2,305.7$ & $1,516.9$ & $3,094.5$ & $* * *$ \\
$12-3$ & $2,347.3$ & $1,483.6$ & $3,211.0$ & $* * *$ \\
$12-0$ & $2,351.0$ & $1,564.6$ & $3,137.5$ & $* * *$ \\
$1-2$ & $1,843.8$ & $1,376.5$ & $2,311.2$ & $* * *$ \\
$1-3$ & $1,885.5$ & $1,300.5$ & $2,470.4$ & $* * *$ \\
$1-0$ & $1,889.2$ & $1,425.9$ & $2,352.5$ & $* * *$ \\
\hline
\end{tabular}


The average LOS and standard deviation of LOS's based on patients in the sample receiving each of the various anticoagulants is given in Table 4.5. It is noted again that observations associated with Acombo treatments 13 and 23 were removed from further consideration because of their small sample sizes. Since it appeared from Table 4.5 that the equal variance assumption might not be met, a regression analysis was conducted with the natural $\log$ of LOS as the dependent variable and the independent variables including the indicator variables for the anticoagulants, gender of the patients, and age of the patients. Residual analysis conducted indicated that the assumptions of the transformed model were fine. Age and gender are significant in the LOS model. Females stay significantly longer than male patients by .13 days on the average. Older patients stay significantly longer, although this is a very small estimated difference per year, 0.0083 days. The anticoagulant given to patients is significantly associated with LOS controlling for the gender of the patient and the age of the patient. The ANOVA table is given in Table 4.6 (a) for the transformed model along with the estimated coefficients for age and gender based on LOS, the untransformed model in Table 4.6 (b).

Table 4.5 LOS Summary Statistics for Acombo

\begin{tabular}{ccc}
\hline Acombo & Mean & Std Dev \\
\hline 0 & 2.48 & 0.99 \\
1 & 2.50 & 1.09 \\
2 & 3.00 & 1.16 \\
3 & 2.80 & 0.85 \\
12 & 3.83 & 3.00 \\
13 & 3.00 & 1.00 \\
23 & 3.36 & 1.63 \\
Overall & 2.76 & 1.31 \\
\hline
\end{tabular}

Note: Three outliers were removed before forming this table.

Table 4.6(a) LOS Variable Regression Summary

\begin{tabular}{|c|c|c|c|c|c|}
\hline Source & $\mathrm{DF}$ & Type III SS & Mean Square & F Value & $\operatorname{Pr}>F$ \\
\hline acombo & 4 & 11.6322 & 2.9080 & 23.69 & $<.0001$ \\
\hline Gender & 1 & 5.0124 & 5.0124 & 40.83 & $<.0001$ \\
\hline Age & 1 & 11.8244 & 11.8244 & 96.32 & $<.0001$ \\
\hline \multicolumn{6}{|c|}{ Table 4.6. (b) Coefficients of Age and Gender } \\
\hline \multicolumn{2}{|c|}{$\overline{\text { Parameter }}$} & Estimate & andard Error & t Value & $\operatorname{Pr}>|t|$ \\
\hline \multicolumn{2}{|c|}{ Intercept } & .4728 & 0.069 & 6.77 & $<.0001$ \\
\hline \multicolumn{2}{|c|}{ Gender M } & 0.00 & . & & . \\
\hline \multicolumn{2}{|c|}{ Gender F } & .1307 & .0204 & 6.39 & $<.0001$ \\
\hline \multicolumn{2}{|c|}{ Age } & 0.0083 & 0.0008 & 9.69 & $<.0001$ \\
\hline
\end{tabular}

Tukey's multiple comparison test was conducted for the various anticoagulants. Comparisons were first conducted using the transformed model with natural log of the number of days as the dependent variable. Tukey's multiple comparisons were also formed on the untransformed data with similar results. The 95\% confidence intervals for the significant differences in LOS are given in Table 4.7. Anticoagulants 12, 2, and 3 are associated with the longest lengths of stay and anticoagulants 0 and 1 are associated with the shortest lengths of stay.

A chi-square test of independence is conducted based on anticoagulants $0,1,2,3$, and 12 and the proportion of early readmissions. No significant differences in the proportions of early readmissions were found $(\mathrm{p}$-value $=0.5191)$. Fischer's exact test was also conducted on the data and no significant differences in the proportions were again found ( $\mathrm{p}$-value $=0.4762$ ).

It should be noted that a possible complication found with the results is that the anticoagulants are confounded with the various hospital locations. Table 4.8 has the number of patients given the various anticoagulants at each location (top line), the percentage of patients at that location given that anticoagulant $\left(2^{\text {nd }}\right.$ line), and the percentage of all the patients given that anticoagulant at that location (bottom line). It is noted that all 9 patients at Location $\mathrm{E}$ are given the same anticoagulant. There are 
102 out of 109 patients at Location B given the same anticoagulant and 310 patients out of 386 at Location D given the same anticoagulant. The different results from the different anticoagulants could just be associated with the differences in the various locations. Because of this, the results associated with Location $\mathrm{C}$ were examined on their own. Location $\mathrm{C}$ had the widest variety of anticoagulant usages. The mean and standard deviation of hospital costs associated with patients in Location $\mathrm{C}$ using the various anticoagulants is given in Table 4.9 .

Table 4.7 Significant LOS Results from Tukey

\begin{tabular}{ccccc}
\hline Acombo Comparison & Days Increase Estimate & 95\% Confidence Interval & .05 level significance \\
\hline $12-3$ & 0.5551 & 0.1758 & 0.9344 & $* * *$ \\
$12-1$ & 0.8481 & 0.4870 & 1.2093 & $* * *$ \\
$12-0$ & 0.8696 & 0.4534 & 1.2858 & $* * *$ \\
$2-1$ & 0.4968 & 0.2726 & 0.7209 & $* * *$ \\
$2-0$ & 0.5183 & 0.2133 & 0.8232 & $* * *$ \\
$3-1$ & 0.2923 & 0.0709 & 0.5152 & $* * *$ \\
$3-0$ & 0.3145 & 0.0109 & 0.6180 & $* * *$ \\
\hline
\end{tabular}

Table 4.8 Location x Acombo Frequency Table

\begin{tabular}{|c|c|c|c|c|c|c|}
\hline \multicolumn{7}{|c|}{ Acombo } \\
\hline Location & 0 & 1 & 2 & 3 & 12 & Total \\
\hline \multirow{3}{*}{ A } & 1 & 3 & 2 & 6 & 1 & \multirow{3}{*}{$\begin{array}{c}13 \\
1.08\end{array}$} \\
\hline & 7.69 & 23.08 & 15.38 & 46.15 & 7.69 & \\
\hline & 0.73 & 0.65 & 0.78 & 2.26 & 1.30 & \\
\hline \multirow{3}{*}{ B } & 0 & 102 & 0 & 0 & 7 & \multirow{3}{*}{$\begin{array}{c}109 \\
9.08\end{array}$} \\
\hline & 0.00 & 93.58 & 0.00 & 0.00 & 6.42 & \\
\hline & 0.00 & 22.03 & 0.00 & 0.00 & 9.09 & \\
\hline \multirow{3}{*}{$\mathrm{C}$} & 112 & 48 & 242 & 247 & 34 & \multirow{3}{*}{$\begin{array}{c}683 \\
56.92\end{array}$} \\
\hline & 16.40 & 7.03 & 35.43 & 36.16 & 4.98 & \\
\hline & 81.75 & 10.37 & 93.80 & 93.21 & 44.16 & \\
\hline \multirow{3}{*}{ D } & 24 & 310 & 5 & 12 & 35 & \multirow{3}{*}{$\begin{array}{c}386 \\
32.17\end{array}$} \\
\hline & 6.22 & 80.31 & 1.30 & 3.11 & 9.07 & \\
\hline & 17.52 & 66.95 & 1.94 & 4.53 & 45.45 & \\
\hline \multirow{3}{*}{$\mathbf{E}$} & 0 & 0 & 9 & 0 & 0 & \multirow{3}{*}{$\begin{array}{c}9 \\
0.75\end{array}$} \\
\hline & 0.00 & 0.00 & 100.00 & 0.00 & 0.00 & \\
\hline & 0.00 & 0.00 & 3.49 & 0.00 & 0.00 & \\
\hline \multirow{2}{*}{ Total } & 137 & 463 & 258 & 265 & 77 & 1200 \\
\hline & 11.42 & 38.58 & 21.50 & 22.08 & 6.42 & 100.00 \\
\hline
\end{tabular}

Note: The first number of each cell is the frequency, the second is the row percent, and the third is the column percent.

Table 4.9 Summary of Cost by Acombo Using Hospital C

\begin{tabular}{ccc}
\hline Acombo & Mean & Std Dev \\
\hline 0 & $\$ 9,602$ & $\$ 1,557$ \\
1 & $\$ 9,944$ & $\$ 2,085$ \\
2 & $\$ 10,104$ & $\$ 1,725$ \\
3 & $\$ 9,827$ & $\$ 1,413$ \\
12 & $\$ 11,499$ & $\$ 4,402$ \\
Overall & $\$ 9,980$ & $\$ 1,886$ \\
\hline
\end{tabular}

A regression model with cost as the dependent variable using only data from Location $\mathrm{C}$ was fitted and the anticoagulants were found to be significant. Both transformed (natural log of the hospital costs) and untransformed data were used with similar results. Tukey's multiple comparison test was conducted on the untransformed data and the significant differences and resulting confidence intervals in differences in costs is given in Table 4.10. 
Table 4.10 Significant Cost Results from Tukey Using Location C

\begin{tabular}{ccccc}
\hline Acombo Comparison & Dollar Increase Estimate & 95\% Confidence Interval & .05 level significance \\
\hline $12-2$ & 1394.2 & 467.0 & 2321.3 & $* * *$ \\
$12-1$ & 1554.0 & 419.3 & 2688.8 & $* * *$ \\
$12-3$ & 1671.1 & 745.1 & 2597.1 & $* * *$ \\
$12-0$ & 1896.1 & 904.9 & 2887.3 & $* * *$ \\
\hline
\end{tabular}

The mean and standard deviation of LOS only at Location C associated with each anticoagulant is given in Table 4.11. Two regression models were formed; one using LOS as the dependent variable (untransformed); and one using natural log of LOS as the dependent variable (transformed), using only the data from Location C. The anticoagulant used was significantly associated with LOS. Tukey's multiple comparison was used on both the transformed and untransformed models with similar results. The resulting significant confidence intervals for the differences in LOS (untransfomed) are given in Table 4.12

Table 4.11 Summary of LOS by Acombo Using Hospital C

\begin{tabular}{ccc}
\hline \multicolumn{3}{l}{ AcomboMeanStd Dev } \\
\hline 0 & 2.64 & 0.95 \\
1 & 2.93 & 1.01 \\
2 & 2.98 & 1.18 \\
3 & 2.82 & 0.81 \\
12 & 3.00 & 3.00 \\
Overall & 2.93 & 1.22 \\
\hline
\end{tabular}

Table 4.12 Significant LOS Results from Tukey Using Location C

\begin{tabular}{ccccc}
\hline Acombo Comparison & Day Increase Estimate & 95\% Confidence Interval & .05 level significance \\
\hline $12-2$ & 0.74 & 0.19 & 1.29 & $* * *$ \\
$12-1$ & 0.78 & 0.12 & 1.44 & $* * *$ \\
$12-3$ & 0.89 & 0.35 & 1.43 & $* * *$ \\
$12-0$ & 1.08 & 0.50 & 1.65 & $* * *$ \\
$2-0$ & 0.34 & 0.01 & 0.67 & $* * *$ \\
\hline
\end{tabular}

A comparison was also conducted to observe any potential differences in RA among the anticoagulants when just Location $\mathrm{C}$ was taken into consideration. A chi-square test and Fisher's exact test were used to test for differences in proportions of early readmissions. The p-values associated with the chi-square test and Fisher's exact test were 0.0693 and 0.0676 , respectively. Although not significant at 0.05, these results do indicate marginal significance. The frequencies of readmissions and nonreadmissions are given in Table 4.13 .

Table 4.13 Re-admittance by Acombo using Location C

\begin{tabular}{ccccccc}
\hline \multicolumn{7}{c}{ Acombo } \\
\hline RA & $\mathbf{0}$ & $\mathbf{1}$ & $\mathbf{2}$ & $\mathbf{3}$ & $\mathbf{1 2}$ & Total \\
\hline \multirow{2}{*}{$\mathbf{0}$} & 111 & 44 & 235 & 242 & 32 & 664 \\
& 99.11 & 6.44 & 34.41 & 35.43 & 4.69 & 97.22 \\
\multirow{2}{*}{$\mathbf{1}$} & $1(.90 \%)$ & $4(9.1 \%)$ & $7(2.6 \%)$ & $5(2.1 \%)$ & $2(6.25 \%)$ & $19(2.86 \%)$ \\
Total & 0.15 & 0.59 & 1.02 & 0.73 & 0.29 & 2.78 \\
\hline
\end{tabular}

Note: The first number in each cell is the frequency; the second number is the column percent.

There were a few differences found between the results from the overall data and the results found by using Location $\mathrm{C}$ only. However, the anticoagulant associated with the largest cost and longest LOS is anticoagulant 12. From examining Table 4.13, we also see that anticoagulant treatment 12 is associated 
with a possible higher proportion of early readmissions (6.25\%). It appears that anticoagulant treatment 1 may also be associated with a higher proportion of early readmissions $(9.1 \%)$.

\subsection{Regional Anesthesia Drugs}

We will next compare the regional anesthesia drugs as to their association with hospital cost and length of stay. The frequency with which each of the local anesthesia drugs were used is given in Table 4.13. The mean cost of the hospital stay and the standard deviation of the costs of the hospital stays associated with the patients using these drugs is given in Table 4.14. Three large outliers were removed before computing means and standard deviations as mentioned in the case of the anticoagulants.

Table 4.13 Frequency Table of Scombo

\begin{tabular}{ccccc}
\hline scombo & Frequency & Percent & Cumulative Frequency & Cumulative Percent \\
\hline 0 & 82 & 6.75 & 82 & 6.75 \\
1 & 78 & 6.43 & 160 & 13.18 \\
2 & 525 & 43.25 & 685 & 56.43 \\
3 & 2 & 0.16 & 687 & 56.59 \\
12 & 519 & 42.75 & 1206 & 99.34 \\
13 & 3 & 0.25 & 1209 & 99.59 \\
23 & 2 & 0.16 & 1211 & 99.75 \\
123 & 3 & 0.25 & 1214 & 100.00 \\
\hline
\end{tabular}

Table 4.14 Cost Summary Statistics by Scombo

\begin{tabular}{ccc}
\hline scombo & Mean & Standard Deviation \\
\hline 0 & $\$ 10,718$ & $\$ 2,358$ \\
1 & $\$ 12,719$ & $\$ 5,398$ \\
2 & $\$ 10,060$ & $\$ 1,728$ \\
3 & $\$ 14,790$ & $\$ 11,788$ \\
12 & $\$ 11,682$ & $\$ 2,900$ \\
13 & $\$ 10,445$ & $\$ 4,195$ \\
23 & $\$ 6,213$ & $\$ 978$ \\
123 & $\$ 11,485$ & $\$ 3,209$ \\
\hline
\end{tabular}

Note: Three outliers were removed before forming this table.

A regression analysis was conducted with the natural log of hospital cost as the dependent variable (to meet the regression assumptions of equal variance and normality) and age of patient, gender of patient, and the indicator variables for the local anesthetics used as the independent variables. Observations associated with regional anesthetics 3, 13, 23, and 123 were removed because of the small sample sizes before conducting the regression analysis, as well as the three large outliers. Gender and age were not significant in the regression model and were removed. The regression model output with the indicator variables for the four local anesthesia drugs is given in Table 4.15. There is a significant association between hospital cost and regional anesthetic drug used. Tukey's multiple comparison was done on the transformed and then untransformed data with similar results. These are given in Table 4.16 ( $\mathrm{a}$ and $\mathrm{b}$ ). Table $4.16 \mathrm{c}$ summarizes the findings.

Table 4.15 Regression Model Output Associated with Hospital Cost (Regional Anesthesia Drugs-Independent Variables)

\begin{tabular}{cccccc}
\hline Source & DF & Sum of Squares & Mean Square & F Value & Pr $>$ F \\
\hline Model & 3 & 5.6520 & 1.8840 & 60.16 & $<.0001$ \\
Error & 1197 & 37.4882 & 0.0313 & & \\
Corrected Total & 1200 & 43.1402 & & & \\
\hline
\end{tabular}

The association between the different regional anesthesia drugs and length of stay was next considered. The average length of stay and the standard deviation of the length of stays associated with each of the 
drugs is given in Table 4.17. A regression analysis is conducted with LOS as the dependent variable and age of patient, gender of patient, and the indicator variable of the regional anesthesia given to the patient in the model as the independent variables. A regression analysis is also conducted with natural $\log$ of LOS as the dependent variable. The regression table output for the transformed model is given in Table 4.18.

Table 4.16(a) Significant Cost Results from Tukey-Transformed Model

\begin{tabular}{ccccc}
\hline Scombo ComparisonPercent Increase Estimate95\% Confidence Interval.05 level significance \\
\hline $1-0$ & $13.18 \%$ & $5.29 \%$ & $21.66 \%$ & $* * *$ \\
$1-2$ & $19.64 \%$ & $13.17 \%$ & $26.47 \%$ & $* * *$ \\
$12-0$ & $8.23 \%$ & $2.53 \%$ & $14.25 \%$ & $* * *$ \\
$12-2$ & $14.40 \%$ & $11.22 \%$ & $17.68 \%$ & $* * *$ \\
$0-2$ & $5.70 \%$ & $0.14 \%$ & $11.58 \%$ & $* * *$ \\
\hline
\end{tabular}

Table 4.16(b) Significant Cost Results from Tukey-Untransformed Model

\begin{tabular}{ccccc}
\hline Scombo ComparisonDollar Increase Estimate95\% Confidence Interval.05 level significance \\
$1-0$ & $1,495.6$ & 588.6 & $2,402.6$ & $* * *$ \\
$1-2$ & $2,153.4$ & $1,455.9$ & $2,850.8$ & $* * *$ \\
$12-0$ & 871.2 & 191.8 & $1,550.6$ & $* * *$ \\
$12-2$ & $1,529.0$ & $1,174.8$ & $1,883.1$ & $* * *$ \\
$0-2$ & 657.8 & -20.9 & $1,336.4$ & \\
\hline
\end{tabular}

Table 4.16 (c) Rank of Scombo by Cost

\begin{tabular}{cc}
\hline Rank & Treatment \\
\hline 1 (highest) & 12,1 \\
2 & 0 \\
3 (lowest) & 2 \\
\hline
\end{tabular}

Table 4.17. LOS Summary Statistics by Scombo

\begin{tabular}{ccc}
\hline scombo & Mean & Standard Deviation \\
\hline 0 & 2.76 & .95 \\
1 & 3.22 & 2.15 \\
2 & 2.89 & 1.05 \\
3 & 7.50 & 4.95 \\
12 & 2.55 & 1.35 \\
13 & 3.00 & 1.00 \\
23 & 3.50 & .71 \\
123 & 2.67 & .58 \\
\hline
\end{tabular}

Note: Three outliers were removed before forming this table.

Table 4.18. Variable Regression Output for LOS

\begin{tabular}{cccccc}
\hline Source & DF & Type III SS & Mean Square & F Value & Pr $>$ F \\
\hline scombo & 3 & 10.2527 & 3.4175 & 27.94 & $<.0001$ \\
Age & 1 & 13.5886 & 13.5886 & 111.11 & $<.0001$ \\
Gender & 1 & 4.1303 & 4.1303 & 33.77 & $<.0001$ \\
\hline
\end{tabular}

Age and gender were significant in the transformed and untransformed LOS models. The coefficients associated with age and gender are given in Table 4.19 based on the untransformed data. Although significant, age does not appear to have much of an effect on LOS. For every 10 years older that a patient is, the estimated increase in LOS on average is about a tenth of a day. Females also are associated with a slightly longer length of stay (.12 days on average). The regional anesthesia used is significantly associated with LOS after controlling for age and gender (p-value<0.0001). Tukey's multiple comparison test was performed on the various regional anesthesia drugs in terms of LOS and 
natural $\log$ of LOS. Results are similar for both the transformed and untransformed models. The significant results are given in Table 4.20 based on the untransformed data. Regional anesthetics 1 and 2 are associated with significantly longer LOS when compared to regional anesthetic 12 by approximately .5 and .4 days, respectively.

Table 4.19. Coefficients for Age and Gender

\begin{tabular}{ccccc}
\hline Parameter & Estimate & Standard Error & $\mathbf{t}$ Value & $\operatorname{Pr}>|\mathbf{t}|$ \\
\hline Intercept & 0.1769 & 0.0575 & 3.07 & 0.0022 \\
Age & 0.0089 & 0.0008 & 10.54 & $<.0001$ \\
Gender F & 0.1184 & 0.0203 & 5.81 & $<.0001$ \\
Gender M & 0.0000 &. &. &. \\
\hline
\end{tabular}

Table 4.20. Significant LOS Results from Tukey-Untransformed Model

\begin{tabular}{ccccc}
\hline Scombo Comparison & Day Increase Estimate & 95\% Confidence Interval & .05 level significance \\
\hline $1-12$ & 0.51009 & 0.18173 & 0.83844 & $* * *$ \\
$2-12$ & 0.38662 & 0.22007 & 0.55317 & $* * *$ \\
\hline
\end{tabular}

We will next test to see if the readmission rate is significantly different between the different regional anesthesia drugs. A chi-square test is conducted with a p-value of 0.9992. There is no evidence to suggest that any of the RA rates associated with the different drugs are significantly different.

It may be that the effects of the regional anesthesia drugs are confounded with location because the different hospitals favor different drugs. Table 4.21 shows the frequency of the different drugs at each of the hospitals. It is noted that Location $\mathrm{D}$ favors Scombo drug 12, as an example. It appears that Location $\mathrm{C}$ has the widest variety of the different Scombo drugs. We will further investigate just the data from Location C. Table 4.22 gives the mean and standard deviation of the hospital costs for patients given each of the various Scombos at Location C. Regression models were formed based on total hospital cost and then the natural log of total hospital cost and then Tukey's multiple comparison test was conducted on both the transformed and untransformed models with similar results. Table 4.24 gives the significant results from Tukey's multiple comparison technique for differences in hospital costs associated with the various local anesthesia drugs. The only significant difference in cost was found between drugs 1 and 2 .

Table 4.23 gives the mean and standard deviation of LOS associated with each of the various regional anesthesia drugs given at Location C. Tukey's multiple comparison test was performed using both the transformed (natural log of LOS) and untransformed (LOS) models and there were no significant results.

Table 4.21 Location x Scombo Frequency Table

\begin{tabular}{|c|c|c|c|c|c|}
\hline \multicolumn{6}{|c|}{ Acombo } \\
\hline Location & 0 & 1 & 2 & 12 & Total \\
\hline \multirow{3}{*}{ A } & 0 & 9 & 0 & 3 & \multirow{3}{*}{12} \\
\hline & 0.00 & 75.00 & 0.00 & 25.00 & \\
\hline & 0.00 & 11.69 & 0.00 & 0.58 & \\
\hline \multirow{3}{*}{ B } & 38 & 16 & 43 & 12 & \multirow{3}{*}{109} \\
\hline & 34.86 & 14.68 & 39.45 & 11.01 & \\
\hline & 46.34 & 20.78 & 8.19 & 2.32 & \\
\hline \multirow{3}{*}{$\mathrm{C}$} & 42 & 31 & 472 & 149 & \multirow{3}{*}{694} \\
\hline & 6.05 & 4.47 & 68.01 & 21.47 & \\
\hline & 51.22 & 40.267 & 89.90 & 28.82 & \\
\hline \multirow{3}{*}{ D } & 1 & 21 & 9 & 350 & \multirow{3}{*}{381} \\
\hline & 0.26 & 5.51 & 2.36 & 91.86 & \\
\hline & 1.22 & 27.27 & 1.71 & 67.70 & \\
\hline \multirow{3}{*}{$\mathrm{E}$} & 1 & 0 & 1 & 3 & \multirow{3}{*}{5} \\
\hline & 20.00 & 0.00 & 20.00 & 60.00 & \\
\hline & 1.22 & 0.00 & 0.19 & 0.58 & \\
\hline Total & 82 & 77 & 525 & 517 & 1201 \\
\hline
\end{tabular}

Note: The first number of each cell is the frequency, the second is the row percent, and the third is the column percent. 
Table 4.22. Cost Summary Statistics by Scombo for Location C

\begin{tabular}{ccc}
\hline Scombo & Mean & Standard Deviation \\
\hline 0 & $\$ 10,718$ & $\$ 2,358$ \\
1 & $\$ 12,214$ & $\$ 3,054$ \\
2 & $\$ 10,060$ & $\$ 1,728$ \\
12 & $\$ 11,638$ & $\$ 2,813$ \\
\hline
\end{tabular}

Table 4.23. LOS Summary Statistics by Scombo for Location $\mathrm{C}$

\begin{tabular}{ccc}
\hline Scombo & Mean & Standard Deviation \\
\hline 0 & 2.756 & 0.950 \\
1 & 3.013 & 1.164 \\
2 & 2.900 & 1.046 \\
12 & 2.503 & 1.154 \\
\hline
\end{tabular}

Table 4.24. Significant Cost Results from Tukey-Untransformed Model

\begin{tabular}{ccccc}
\hline Scombo Comparison & Dollar Increase Estimate & 95\% Confidence Interval & .05 level significance \\
\hline $1-2$ & 1084.8 & 226.3 & 1943.3 & $* * *$ \\
\hline
\end{tabular}

\subsection{Blood Transfusion Prevention Drug}

The last drug category to be considered is the category of drugs designed to prevent blood transfusions. There was only one drug considered in this category that was possibly given to patients, tranexamic acid. We will define a variable, b1, to equal 0 if a patient did not receive this drug and 1 if a patient received this drug. Table 4.25 lists the frequency of patients given the drug and not given the drug and then whether or not they needed a blood transfusion. A chi-square test is conducted and it was found that the proportion of those patients needing a blood transfusion is significantly higher for patients not receiving the drug $(\mathrm{p}$-value $<0.0001)$. A logistic regression was also conducted with the dependent variable being 1 if the patient needed a blood transfusion (BT) and 0 otherwise with the indicator variable, b1, for tranexamic acid in the model while controlling for both age and gender. Point estimates and associated confidence intervals for the logistic regression model are given in Table 4.26. The odds of needing a blood transfusion are estimated to be 4 times higher for patients not receiving tranexamic acid. The odds of females needing a blood transfusion are estimated to be 2.7 times the odds of males needing a blood transfusion. The odds of needing a blood transfusion also increase slightly with age, with the odds getting multiplied by 1.033 for each additional year that a person ages.

Table 4.25. Frequency Table for b1 x Blood Transfusion

\begin{tabular}{cccc}
\hline \multicolumn{4}{c}{ Blood Transfusion } \\
\hline b1 & $\mathbf{0}$ & $\mathbf{1}$ & Total \\
$\mathbf{0}$ & 536 & 132 & 668 \\
$\mathbf{1}$ & 512 & 34 & 546 \\
Total & 104 & 166 & 1214 \\
\hline
\end{tabular}

Table 4.26. Logistic Regression for BT $=1$

\begin{tabular}{cccc}
\hline Effect & Point Estimate & 95\% Wald Confidence Limits \\
\hline b1 0 vs 1 & 4.000 & 2.668 & 5.996 \\
Age & 1.033 & 1.018 & 1.049 \\
Gender F vs M & 2.720 & 1.875 & 3.945 \\
\hline
\end{tabular}

Hospital cost associated with whether or not a patient had tranexamic acid was also considered. The sample mean and sample standard deviation of hospital costs associated with patient who did not receive tranexamic acid $(\mathrm{b} 1=0)$ and those patients that did receive tranexamic acid $(\mathrm{b} 1=1)$ were calculated and are given in Table 4.27. Regression models were formed with hospital cost 
(untransformed) and then natural log of hospital cost (transformed) as the dependent variables with the independent variable being whether or not the patients were given tranexamic acid. Patients who had tranexamic acid were significantly associated with a higher hospital cost. The ANOVA table based on the transformed data is given in Table 4.28. It was found that the amount of increase in cost associated with these patients was between $\$ 811$ and $\$ 1341$.

LOS associated with whether or not a patient had tranexamic acid was next considered. Patients who had not been given the drug had an average LOS of 2.97 days with a standard deviation of 1.20 days. Patients who had been given the drug had an average LOS of 2.43 days with a standard deviation of 0.96 days. A regression analysis was conducted with LOS being the dependent variable with the indicator variable of whether or not the patient had been given tranexamic acid put into the model while controlling for age and gender. It was found that tranexamic acid was associated with a shorter length of stay ( $\mathrm{p}$-value < 0.0001). A 95\% confidence interval was calculated between the LOS for those patients given the drug and those not given the drug and found to be -.73 to -.46, so on the average patients got out of the hospital about $1 / 2$ to $3 / 4$ day sooner if they were given the drug.

The proportions of patients given early readmission between those given tranexamic acid and those not given the drug were considered. A chi-square test was performed and no significant difference was found (p-value=.9553).

Table 4.27. Cost Summary Statistics by b1

\begin{tabular}{ccc}
\hline b1 & Mean & Standard Deviation \\
\hline 0 & $\$ 10,479$ & $\$ 2,939$ \\
1 & $\$ 11,582$ & $\$ 2,607$ \\
\hline
\end{tabular}

Note: Three outliers were removed before forming this table.

Table 4.28. Regression Model of Cost

\begin{tabular}{cccccc}
\hline Source & DF & Sum of Squares & Mean Square & F Value & Pr $>$ F \\
\hline Model & 1 & 3.4551 & 3.4551 & 88.75 & $<.0001$ \\
Error & 1212 & 47.1814 & 0.0389 & & \\
Corrected Total & 1213 & 50.6365 & & & \\
\hline
\end{tabular}

Location might have been confounded with cost, LOS, and early readmissions. Because of this, Location $\mathrm{C}$ only was considered. There were 531 patients at this location not given tranexamic acid and 166 patients who were. The mean hospital cost of those patients not given the drug was $\$ 9,976$ with a standard deviation of $\$ 1,909$. The mean hospital cost of those patients given the drug was $\$ 9,849$ with a standard deviation of $\$ 1,010$. The difference in cost was not found to be significant ( $\mathrm{p}$-value $=0.6220$ ).

The LOS of those patients not given the drug at Location $\mathrm{C}$ was 2.96 days with a standard deviation of 1.16 days. The average LOS of those given the drug at Location $\mathrm{C}$ was 2.73 days with a standard deviation of 0.74 days. Patients given the drug had an estimated average shorter length of stay by .24 days (p-value $<0.05)$.

\section{Conclusion}

In our study, we did find that within the different classifications of drugs there was an association between the drug used and the cost and LOS. We cannot say that the drug used cause the difference in cost or LOS, but only that there was an association. In Table 5.1, we list the anticoagulants that are associated with the highest and lowest costs, and then longest and shortest LOS. The combination of warfarin and enoxaparin is significantly associated with the highest hospital cost and longest length of stay. We also found that administering no anticoagulant was associated with the lowest cost and shortest length of stay. Our study did not take into account the occurrence of DVT which may possibly be higher among patients who did not receive an anticoagulant. When we did take into account what was occurring at Location $\mathrm{C}$ only, the anticoagulant combination of warfarin and enoxaparin was still significantly associated with the highest cost and longest LOS, however not receiving an anticoagulant was now only significantly lower in terms of cost and LOS than receiving enoxaparin. A marginally significant difference was found between the proportions of early readmissions among the different 
anticoagulants used when considering only Location $\mathrm{C}$ data ( $\mathrm{p}$-value=0.0693). It appeared that warfarin and the combination of warfarin and enoxaparin had the largest proportions of early readmissions.

Table 5.1. Anticoagulant Ranks in Terms of Cost and LOS

\begin{tabular}{ccc}
\hline Acombo & Cost Rank (1=highest) & LOS (1=highest) \\
\hline Warfarin, Enoxaparin & 1 & 1 \\
Warfarin & 1 & 2 \\
Enoxaparin & 2 & 1 \\
Rivaroxaban & 2 & 1 \\
No Anticoagulant & 2 & 2 \\
\hline
\end{tabular}

The results from considering the associations of hospital costs and LOS association with the different regional anesthetics given are found in Table 5.2. Lidocaine was associated with the highest cost and longest LOS. From past studies, lidocaine was associated with the highest TNS occurrence. There was no clear overall best regional anesthetic in terms of both cost and LOS. Some of the regional anesthetics were associated with lower cost, but longer LOS while one combination (lidocaine and bupivacaine) was associated with a high cost and a shorter length of stay. There were no significant differences in the proportions of early readmissions associated with each of the regional anesthetic drugs used ( $\mathrm{p}$-value $=$ 0.9992). When considering only Location $\mathrm{C}$ data, we only found a significant difference in cost between using lidocaine (highest) and bupivacaine (lowest). We did not find any significant differences in LOS when using Location $\mathrm{C}$ only associated with the different regional anesthesia drugs.

Table 5.2. Regional Anethesia Ranks in Terms of Cost and LOS

\begin{tabular}{ccc}
\hline Scombo & Cost Rank (1=highest) & LOS (1=highest) \\
\hline Lidocaine & 1 & 1 \\
Lidocaine, Bupivacaine & 1 & 2 \\
No Regional Anesthesia & 2 & 1 \\
Bupivacaine & 3 & 1 \\
\hline
\end{tabular}

When analyzing the results for tranexamic acid, we found a significantly higher cost and significantly shorter LOS associated with this drug when the whole data set was considered. No significant difference was found in the proportions of early readmissions for those patients given tranexamic acid and those patients not given tranexamic $\operatorname{acid}(\mathrm{p}$-value $=0.9553)$. There was a significant reduction in the proportion of patients requiring a blood transfusion when given this drug. When considering only data associated with Location C, a significantly shorter LOS was associated with the drug. There was not a significant increase in cost.

Future research could involve considering other types of common medical procedures done in hospitals and testing for any association between the various drugs used in one category with LOS and total hospital cost. Tests across several drug categories could also be considered.

\section{References}

1. Pfuntner, A, LM Wier, and C Steiner. Costs for Hospital Stays in the United States, 2010. HCUP Statistical Brief \#146. Agency for Healthcare Research and Quality, Jan. 2013.

2. $\quad$ Foran, Jared R.H., MD. "Total Hip Replacement." AAOS. N.p., Dec. 2011. Web. 05 May 2015.

3. Wolford, Monica L., M.A., and Anita Bercovitz, M.P.H. "Hospitalization for Total Hip Replacement Among Inpatients Aged 45 and Over: United States, 2000-2010." Centers for Disease Control and Prevention. Centers for Disease Control and Prevention, 12 Feb. 2015. Web. 05 May 2015.

4. Txertsvadze, A., Grove, A., Freeman, K., Court, R., Johnson, S., Connock, M., Clarke, A., Sutcliffe, P. (2014). "Total Hip Replacement for the Treatment of End Stage Arthritis of the Hip: A Systematic Review and Meta-Analysis", PLOS ONE, Web 8 July, 2014 DOI: 10.1371/journal.pone.0099804.

5. Cowley, D. (1995). "Prostheses for Primary Total Hip Replacement: A Critical Appraisal of the Literature", International Journal of Technology Assessment in Health Care, 11(4), 770-779. 
6. Kelly, E., Campbell, J., Murray, P. (2013). "Total hip replacement: patient satisfaction and early outcomes", International Jouranl of Health Care Quality Assurance, 26(3), 262-268.

7. Robold, L. and Bauer, P. (2005). "Yoga and Hip Replacement Surgery", International Journal of Yoga Therapy, 15(1), 65-80.

8. Syryla, W., Pogorzala, A., Rogala, P., Zurawski, P. (2009). "Functional Assessment after Total Hip Replacement, International Journal of Rehabilitation Research, 32(1), 59.

9. Grant, S., St. John, W., Patterson, E. (2009). "Recovery from Total Hip Replacement Surgery: "It's Not Just Physical” “. Qualitative Health Research, 19(11), 1612-1620.

10. American Academy of Orthopaedic Surgeons. "Deep Vein Thrombosis." AAOS. N.p., Jan. 2009. Web. 05 May 2015.

11. "Venous thromboprosphylaxis in general surgery ward admissions: strategies for improvement", International Journal for Quality in Health Care, 2012, 24(6), 649-656.

12. Wera, Glenn D., MD, Ryan M. Garcia, MD, and Victor M. Goldberg, MD. "Reducing Perioperative Bleeding with Antifibrinolytics." AAOS. N.p., Feb. 2013. Web. 05 May 2015.

13. D'Ambrosia, R. D., P. R. Lipscomb, and E. J. McClain. "Prophylactic Anticoagulation in Total Hip Replacement." National Center for Biotechnology Information. U.S. National Library of Medicine, Apr. 1975. Web. 05 May 2015.

14. Eriksson, Bengt I., Lars C. Borris, Richard J. Friedman, Sylvia Haas, Menno V. Huisman, Ajay K. Kakkar, and Tiemo J. Bandel. "Rivaroxaban versus Enoxaparin for Thromboprophylaxis after Hip Arthroplasty." New England Journal of Medicine 358.26 (2008): 2765-775. Web.

15. Gomez-Outes, A., A. I. Terleira-Fernandez, M. L. Suarez-Gea, and E. Vargas-Castrillon. "Dabigatran, Rivaroxaban, or Apixaban versus Enoxaparin for Thromboprophylaxis after Total Hip or Knee Replacement: Systematic Review, Meta-analysis, and Indirect Treatment Comparisons." Bmj 344.Jun14 1 (2012): E3675. Web.

16. Eisenberg, John M., Andrea Humphries, Ph.D, Amelia Williamson Smith, M.S., and Kim Farina, Ph.D. "Preventing Blood Clots After Hip or Knee Replacement Surgery or Surgery for a Broken Hip: A Review of the Research for Adults." AHRQ Effective Health Care Program. N.p., Mar. 2012. Web. 05 May 2015.

17. Mauermann, William J., Ashley M. Shilling, and Zhiyi Zuo. "A Comparison of Neuraxial Block Versus General Anesthesia for Elective Total Hip Replacement: A Meta-Analysis." Anesthesia \& Analgesia 103.4 (2006): 1018025. Web.

18. Rodgers, Anthony, and Natalie Walker. "Reduction of Postoperative Mortality and Morbidity with Epidural or Spinal Anaesthesia: Results from Overview of Randomised Trials." TheBjm. BMJ 2000;321:1493, 16 Dec. 2000. Web.

19. Thorburn, J., J. R. Louden, and R. Vallance. "Spinal And General Anaesthesia In Total Hip Replacement: Frequency Of Deep Vein Thrombosis." BJA: British Journal of Anaesthesia 52.11 (1980): 1117-121. Web.

20. NYSORA. "Spinal Anesthesia." NYSORA. The New York School of Regional Anesthesia, 10 Apr. 2013. Web. 03 May 2015.

21. Zaric, Dusanka, and Nathan L. Pace. "Transient Neurologic Symptoms (TNS) following Spinal Anaesthesia with Lidocaine versus Other Local Anaesthetics." The Cochrane Library. John Wiley \& Sons, Ltd., 15 Apr. 2008. Web. 03 May 2015.

22. Hampl, Karl F., and Sidonie Heinzmann-Wiedmer. "Transient Neurologic Symptoms after Spinal Anesthesia : A Lower Incidence with Prilocaine and Bupivacaine than with Lidocaine." Anesthesiology. Lippincott-Raven Publishers, Mar. 1998. Web.

23. Zaric, Dusanka, Christian Christiansen, Nathan L. Pace, and Yodying Punjasawadwong. "Transient Neurologic Symptoms After Spinal Anesthesia with Lidocaine Versus Other Local Anesthetics: A Systematic Review of Randomized, Controlled Trials." Anesthesia \& Analgesia 100.6 (2005): 1811-816. Web.

24. Bertini, L., S. Mancini, P. Di Benedetto, A. Ciaschi, O. Martini, S. Nava, and V. Tagariello. "Postoperative Analgesia by Combined Continuous Infusion and Patient-controlled Epidural Analgesia (PCEA) following Hip Replacement: Ropivacaine versus Bupivacaine." Acta Anaesthesiologica Scandinavica 45.6 (2001): 782-85. Web.

25. Mcnamee, D. A., A. M. Mcclelland, S. Scott, K. R. Milligan, L. Westman, and U. Gustafsson. "Spinal Anaesthesia: Comparison of Plain Ropivacaine $5 \mathrm{Mg}$ Ml-1 with Bupivacaine $5 \mathrm{Mg} \mathrm{Ml-1}$ for Major Orthopaedic Surgery." British Journal of Anaesthesia 89.5 (2002): 702-06. Web. 
26. Wulf, H., and J. Biscoping. "Ropivacaine Epidural Anesthesia and Analgesia Versus General... : Anesthesia \& Analgesia." LWW. International Anesthesia Research Society, July 1999. Web. 03 May 2015.

27. Bower, Wendy F., Lawrence Jin, and Malcolm J. Underwood. "Peri-operative Blood Transfusion Increases Length of Hospital Stay and Number of Postoperative Complications in Non-cardiac Surgical Patients | HKMJ." Peri-operative Blood Transfusion Increases Length of Hospital Stay and Number of Postoperative Complications in Non-cardiac Surgical Patients | HKMJ. Hong Kong Med J, Apr. 2010. Web. 04 May 2015.

28. Poeran, Jashvant, Rehana Rasul, and Suzuko Suzuki. "Tranexamic Acid Use and Postoperative Outcomes in Patients Undergoing Total Hip or Knee Arthroplasty in the United States: Retrospective Analysis of Effectiveness and Safety." TheBmj. BMJ, 17 July 2014. Web. 04 May 2015.

29. Johansson, T., LG Pettersson, and B. Lisander. "Tranexamic Acid in Total Hip Arthroplasty Saves Blood and Money: A Randomized, Double-blind Study in 100 Patients." National Center for Biotechnology Information. U.S. National Library of Medicine, June 2005. Web. 04 May 2015.

30. Gandhi, Rajiv, Heather Mk Evans, Safiyyah R. Mahomed, and Nizar N. Mahomed. "Tranexamic Acid and the Reduction of Blood Loss in Total Knee and Hip Arthroplasty: A Meta-analysis." BMC Research Notes 6.1 (2013): 184. Web.

31. Irisson, E., Y. Hémon, V. Pauly, S. Parratte, J.-N. Argenson, and F. Kerbaul. "Tranexamic Acid Reduces Blood Loss and Financial Cost in Primary Total Hip and Knee Replacement Surgery." Orthopaedics \& Traumatology: Surgery \& Research 98.5 (2012): 477-83. Web.

32. Panchmatia, Jaykar R., M.A., Soudeh Chegini, B.A., and Charlotte Lobban, M.B. "The Routine Use of Tranexamic Acid in Hip and Knee Replacements." National Center for Biotechnology Information. U.S. National Library of Medicine, 2012. Web. 05 May 2015.

33. Abraham, Bovas, and Johannes Ledolter. Introduction to Regression Modeling. 1st ed. Belmont, CA: Thomson Brooks/Cole, 2006. Print.

34. Kleinbaum, David, Lawrence Kupper, Keith Muller, and Azhar Nizam. Applied Regression Analysis and Multivariable Methods. 3rd ed. New York, NY: Duxbury Press, 1998. Print.

35. Montgomery, Douglas C. Design and Analysis of Experiments. 8th ed. New York: John Wiley \& Sons, 2013. Print.

36. Pagano, Marcello, and Kimberlee Gauvreau. Principles of Biostatistics. 2nd ed. Pacific Grove, CA: Duxbury, 2000. Print.

37. Daniel, Wayne W. Applied Nonparametric Statistics. Australia: Duxbury/Thomson Learning, 1990. Print.

38. Hosmer, David W., and Stanley Lemeshow. Applied Logistic Regression. 2nd ed. New York: Wiley, 19 